\title{
Comparison of Different Algorithms in the Radiotherapy Plans of Breast Cancer
}

\author{
Yonca Yahşi Çelen ${ }^{1}$ and Atilla Evcin ${ }^{2}$ \\ ${ }^{1}$ Department of Radiation Oncology, University of Kocatepe, 03200, Afyon, Turkey \\ ${ }^{2}$ Department of Materials Science and Engineering, University of Kocatepe, 03200, Afyon, Turkey
}

\begin{abstract}
It is aimed to evaluate portal dosimetry results of planned breast cancer patients with intensity-modulated radiotherapy (YART) of Anisotropic Analytical Algorithm (AAA) and Pencil Beam Convolution (PBC) dose calculation algorithms. The plans of 10 treated patients will receive $6 \mathrm{MV}$ photon energy and a total of 25 fractions of 50 Gray dose using the inverse YART technique, which is reverse planned in the Eclipse (ver.13.6) treatment planning system with Varian Trilogy Linear Accelerator prescribing. For each plan, dose was calculated after optimization using PBC and then AAA algorithms. The quality controls of the plans were made using the Electronic Portal Imaging Device (EPID) by creating individual verification plans for each algorithm. In addition, the maximum and average dose values in the target volume were compared in inverse YART plans calculated using $\mathrm{PBC}$ and AAA. When treatment plans generated by $\mathrm{AAA}$ and $\mathrm{PBC}$ dose calculation algorithms are analyzed using EPID, for the PBC algorithm, the mean values of $\mathrm{Y}_{\text {Area }}$ and $\gamma_{\text {Avg }}$ are $98.15 \pm 1.07,0.40 \pm 0.048$ and $98.72 \pm 1.13,0.37 \pm 0.051$, respectively, for the AAA algorithm. The PTV $\mathrm{D}_{\max }$ value for the PBC algorithm is $109.3 \pm 1.09$ and the $\mathrm{D}_{\text {Avg }}$ value is $101.7 \pm 0.51$. For the AAA algorithm, the PTV $D_{\max }$ value is $110.6 \pm 1.12$ and the $D_{\text {Avg }}$ value is 102.9 \pm 0.62 . When the mean values of portal dosimetry $\mathrm{\gamma}$ Area and $\gamma_{\text {Avg }}$ evaluated using PBC and AAA algorithms were compared, the differences between the algorithms were not statistically significant $(p>0.05)$. Differences between the algorithms for PTV $D_{\max }$ and $\mathrm{D}_{\mathrm{Avg}}$ values are not statistically significant ( $\mathrm{p}>0.05)$.
\end{abstract}

\section{Introduction}

Cancer disease is one of the most common health problems in recent years. Many patients with cancer have radiotherapy with or without chemotherapy. Radiotherapy is a method used in the treatment of cancer and some benign diseases using ionizing radiation. The success of radiation therapy is directly related to how well we can maintain robust tissue in addition to controlling tumor volume by irradiating the target. 
External radiotherapy is calculated by dose calculation algorithms found in the treatment planning system (TPS), and the dose calculated by the system's loaded beam data is considered to be close to the actual dose distribution.

The calculated dose, the slight or even different occurrence from the dose administered to the patient, is related to the inhomogeneous dosimetry methods of the dose calculation algorithms.

In general, treatment plans are evaluated on the basis of dose calculations. Some comparisons between calculation algorithms may be found in the literature on breast irradiation, for $\mathrm{PBC}$ and AAA $[1,2,3]$. The calculations using the $\mathrm{PBC}$ algorithm are known to be inaccurate in regions of electronic non-equilibrium, such as in air cavities or in buildup regions, and inhomogeneity corrections due to the lung media or irregular body contour, which are major issues in breast RT [4,5,6]. Monte Carlo (MC) simulation methods and techniques are widely reported for their dose calculation accuracy. Applying $\mathrm{MC}$ techniques to dose calculations in RT has therefore the potential to decrease the uncertainties when compared to analytical/conventional treatment planning algorithms, regardless the beam geometry and target composition [7].

Accurate dose calculation algorithms play an important role in the decrease of global uncertainty and to achieve clinically effective dose distribution for tumor control [8]. Several commercial radiotherapy treatment planning systems (TPS) are implemented with photon beam models that rely on semi-empirical methods, using pencil beam algorithms (PBC) to calculate the absorbed dose and some approximations to correct for tissue inhomogeneities [9].

The aim of this study is to evaluate the results of portal dosimetry of dose calculation algorithms of AAA and PBC dosing in breast cancer patients planned with YART.

\section{Fundamental Properties of the Method}

As shown in figure 1, the plans of 10 treated patients were prepared by using a Varian Trilogy Linear Accelerator device in the Eclipse (ver.13.6) treatment planning system to prescribe $6 \mathrm{MV}$ photon energy and a total of 25 fractions and 50 Gray doses using the inverse planned intensity radiotherapy (Inverse YART) technique. As shown in figure 2, for each plan, dose was calculated after optimization using PBC and then AAA algorithms. The quality controls of the plans were made using the Electronic Portal Imaging Device (EPID) by creating individual verification plans for each algorithm. Gamma analysis was performed based on 3\% dose difference (DD), $3 \mathrm{~mm}$ distance adaptation (DTA) and 95\% data transfer criteria. A $10 \%$ area of isodose was filtered in order to regulate the isodose differences resulting from fiber movements in the off-field low dose regions. In addition, the maximum and average dose values in the target volume were compared in inverse IMRT plans calculated using PBC and AAA.

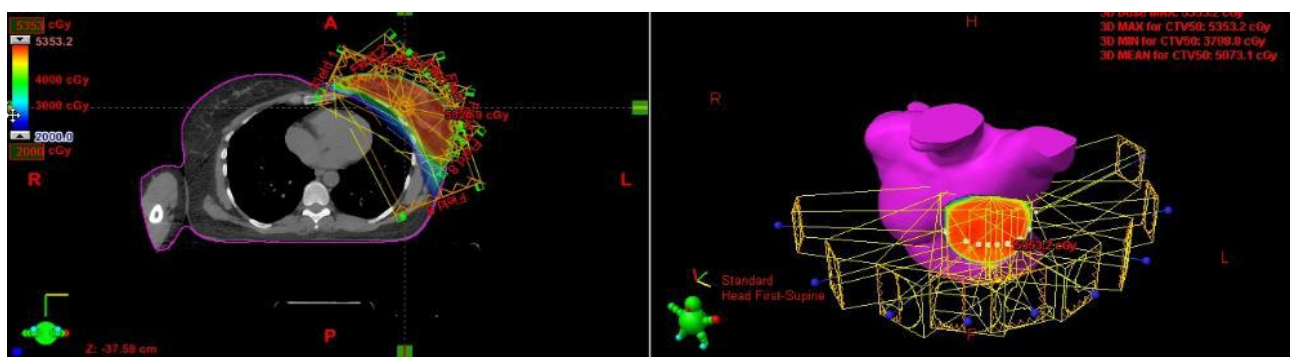

Fig.1. Treatment areas of planned breast cancer patient with YART technique. 


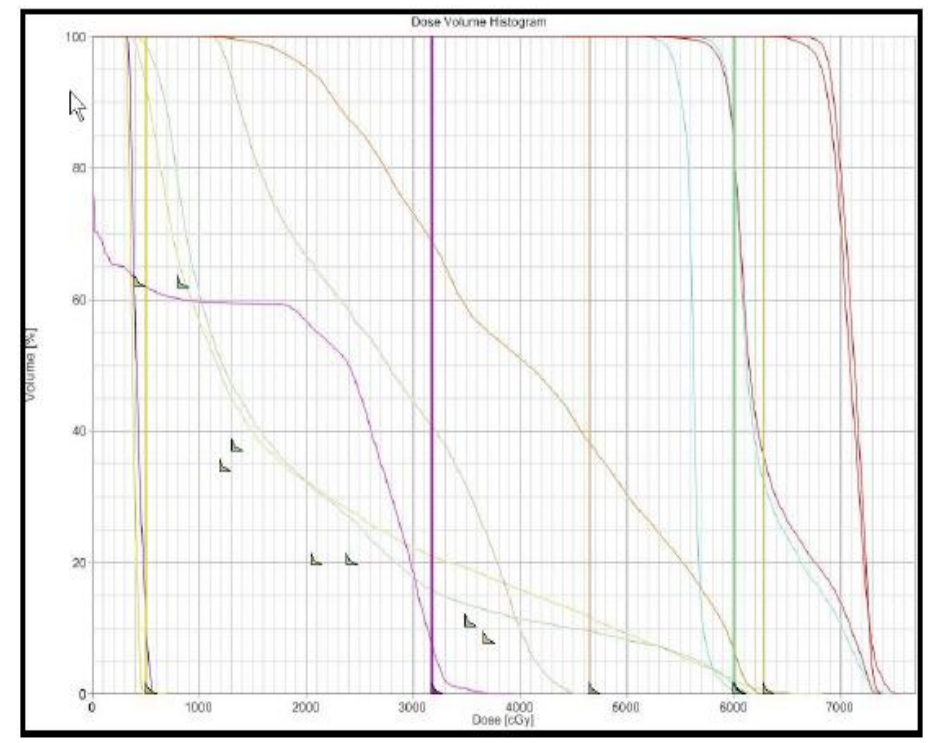

Fig.2. Dose-volume histogram during optimization

\section{Application}

When the treatment plans generated by AAA and $\mathrm{PBC}$ dose calculation algorithms are analyzed using EPID, For the PBC algorithm, as shown in table 1, the mean values of yArea and $\gamma A v g$ are $98.15 \pm 1.070 .40 \pm 0.048$ and $98.72 \pm 1.130 .37 \pm 0.051$, respectively, for the AAA algorithm. The PTV Dmax value for the PBC algorithm is $109.3 \pm 1.09$ and the $\mathrm{D}_{\text {Avg }}$ value is $101.7 \pm 0.51$. For the AAA algorithm, the PTV Dmax value is $110.6 \pm 1.12$ and the $\mathrm{D}_{\mathrm{Avg}}$ value is $102.9 \pm 0.62$.

Table 1. Measurement results of $P B C$ and AAA dose calculation algorithm data with EPID

\begin{tabular}{|c|c|c|}
\hline & PBC & AAA \\
\hline yArea & $98,15 \pm 1,07$ & $98,72 \pm 1,13$ \\
\hline yAvg & $0,40 \pm 0,048$ & $0,37 \pm 0,051$ \\
\hline PTV D $D_{\text {max }}$ & $109,3 \pm 1,09$ & $110,6 \pm 1,12$ \\
\hline PTV D Avg $_{1}$ & $101,7 \pm 0,51$ & $102,9 \pm 0,62$ \\
\hline
\end{tabular}




\section{Conclusion}

Modern computerized planning systems and radiotherapy plans can be created with the dose calculation algorithms closest to the patient's satisfaction. These algorithms can be correction / measurement based, model based or Monte Carlo based. Percent dose, dose profiles and yield factor measurements should be made in water phantom for regular treatment areas under reference conditions to calculate doses in photon energies at Eclipse TPS. Some correction factors such as patient's illness, lack of tissue, tissue inhomogeneity are found by joining the calculator. Dose calculation algorithms use dose calculations and dose profiles taken at various depths by interpolating between deep doses measured in water phantom (8).

Research and development studies are continuing to obtain the current and modern state of the algorithm. AAA requires basic physical parameters to characterize the beam to obtain a dose calculation. These parameters are created by introducing to the TPS the beam data measured by the user for the configuration. The functional form of the basic physical parameters is activated by analytic convolution in AAA.

The AAA algorithm has significant advantages in terms of dose modeling in heterogeneous tissues. In dosing calculations, dose calculations also add to the dose distribution the effects of bundle and bunch-limiting accessories such as block, physical wedge filter, dynamic wedge filter, multi-leaf collimator, tray, and bolus and treatment table (9).

Treatment areas of breast cancer patients radiotherapy include different density tissues such as lung, fat, muscle. Inhomogeneity correction is performed to obtain the dose distribution closest to the truth in radiotherapy plans containing inhomogeneous tissues. Inhomogeneity correction with different dose calculation algorithms such as PBC, AAA and Acuros XB is done by different methods. The PBC algorithm performs inhomogeneity correction using correction methods such as Batho, Modified Batho and Etar. The AAA algorithm involves dose distribution by separately calculating contributions from primary photons, extra focal photons, and contaminant electron sources.

When the mean values of portal dosimetry, $\gamma$ Area and $\gamma$ Avg evaluated using PBC and AAA algorithms were compared, the differences between the algorithms were not statistically significant $(p>0,05)$. Differences between the algorithms for PTV Dmax and $D_{\text {Avg }}$ values are not statistically significant $(\mathrm{p}>0.05)$. 


\section{References}

1. Ahnesjö, M. Saxner, A. Trepp, Med Phys. 19(2), 263e73 (1992)

2. S. Yoo, Q. Wu, J. O’Daniel, J. Horton, F. Yin, Radiother Oncol 103, 172e7 (2012)

3. R. Chakarova, M. Gustafsson, A. Bäck, N. Grugge, A. Palm, A. Lindberg, Radiother Oncol, 102, 102e7 (2012)

4. T. Knöos, A. Ahnesjö, P. Nilsson, P. Weber, Phys. Med. Biol., 40, 1411e20 (1995)

5. V. Panettieri, P. Barsoum, M. Westermark, L. Brualla, I. Lax, Radiother Oncol, 93, 94e101 (2009)

6. S. Almberg, T. Lindmo, J. Frengen, Radiother Oncol, 100, 259e64 (2011)

7. N. Reynaert, S. Marck, D. Schaar, W. Van der Zee, C. Van VlietVroegindeweij, M. Tomsej, Rad. Phys. Chem., 76, 643e86 (2007)

8. N. Papanikolaou, J. Battista, A. Boyer, C. Kappas, E. Klein, T. R. Mackie, Medical Physics Publishing, 2004.

9. M. Siggel, P. Ziegenhein, S. Nill, U. Oelfke, Phys. Med., 28(4), 273e80(2012) 